moment où la recherche en génétique humaine se développe rapidement, où les enjeux scientifiques, mais aussi technologiques et économiques de ce travail sont très importants, à un tel moment donc, l'information est une denrée précieuse. Cela d'autant plus qu'en ma qualité de scientifique travaillant dans ce domaine, je peux avoir accès, à travers des interlocuteurs que je connais déjà personnellement pour la plupart, à une information précise, détaillée et actuelle. Comme par ailleurs j'ai organisé ma disponibilité pour consacrer une année entière au recueil, à l'analyse et à la transmission de cette information, les conditions d'un travail sérieux me semblent réunies. Ce travail, je veux évidemment qu'il soit utilisable par la communauté scientifique française, et c'est bien ainsi que l'entendent les organismes qui les soutiennent : je prévois donc toute une série de "produits" pour assurer la transmission des informations recueillies : depuis le plus analytique (fiches par laboratoire, donnant une idée des travaux poursuivis, des orientations futures, de l'ambiance...) jusqu'au plus global (ouvrage de synthèse sur l'ensemble de l'enquête) en passant par des synthèses intermédiaires sur un sous-domaine, une région ou un pays... et bien sûr par des chroniques génomiques régulières : si j'ai commencé à les tenir au début 1990, c'était aussi pour planter le décor et donner les grandes lignes du domaine que je vais explorer de façon beaucoup plus "pointue " et approfondie en 1991. Sur ce point des produits je suis d'ailleurs très demandeur d'indications sur les désirs des équipes françaises et le type d'information qui leur serait le plus utile : ce n'est qu'en connaissant le mieux possible cette demande que je pourrai maximaliser l'utilité de mon enquête.

J'espère ainsi, à travers ces investigations, arriver à une idée plus précise de la recherche dans ce domaine, de ses enjeux, de ses succès mais aussi des goulots d'étranglement et des points de blocage. Cette étude sera ainsi un des éléments d'une réflexion visant à mieux définir les moyens et les formes d'organisation qui permettront à la recherche française de jouer son rôle dans cet important domaine ; elle aidera aussi à préciser les "créneaux "sur lesquels nous pourrions être les plus performants compte tenu des forces et des faiblesses de notre système

\section{Bertrand Jordan}

Directeur de recherche au Cnrs, responsable du groupe génétique moléculaire humaine, CIML, Inserm/Cnrs, case 906, 13288 Marseille Cedex 9, France.

\title{
FLASH
}

\section{un GĖNE CONTRÔLANT LE POTENTIEL MÉtAstATIQUE DES CELLULES CANCÉREUSES ?}

Marie-Lise Lacombe et al., dans l'équipe de Michel Veron (Institut Pasteur, Paris), viennent de montrer que la nucléotide diphosphate kinase (NDP kinase) dont ils ont cloné un gène chez Dictyostélium discoideum est aussi le produit de gènes étudiés par ailleurs, l'un parce qu'il

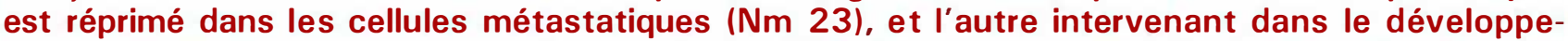
ment des ailes chez la drosophile (Awd). La NDP kinase peut moduler la transduction des signaux empruntant la voie des récepteurs couplés aux G-protéines en fournissant à celles-ci le GTP provoquant leur activation : elle peut aussi contrôler la croissance des microtubules. M.-L. Lacombe, X. Sastre et M. Veron expliqueront, dans un prochain numéro de $\mathrm{m} / \mathrm{s}$, comment, du fait de ces activités biologiques, la NDP kinase peut intervenir dans le potentiel prolifératif de cellules cancéreuses et intervenir dans le développement.

1. Lacombe ML, et al. J Biol Chem $1990 ; 265: 10012-8$.

2. Liotta LA, Steeg PS. J Natl Cancer Inst $1990 ; 82: 1170-2$.

3. Wallet $V$, et al. J Natl Cancer Inst $1990 ; 82: 1199-202$. 